\title{
FLUORESCENCE SPECTROSCOPIC INVESTIGATIONS OF RHODAMINE DYE VAPORS
}

\author{
J. SCHMIDT and A. PENZKOFER \\ Naturwissenschaftlichen Fakultät II, Physik, Universität Regensburg, D-8400 Regensburg, FRG
}

Received 21 November 1988

\begin{abstract}
Rhodamine 19 is heated in a vapor cell and the fluorescence emission is analysed. Above $320^{\circ} \mathrm{C}$ rhodamine 19 vapor disintegrates quickly into 2,7-dimethyl-rhodamine 110 vapor. The fluorescence quantum distribution, the fluorescence quantum efficiency, the stimulated emission cross section and the fluorescence depolarization of this 2,7-dimethyl-rhodamine 110 dye vapor are determined.
\end{abstract}

\section{Introduction}

Spectroscopic studies of rhodamine dye vapors are scarce because these ionic molecules have a very small vapor pressure and their thermal stability is weak at elevated temperatures. Rhodamine 6G [1] and rhodamine B [2] dyestuff was evaporated and analysed by absorption and emission spectroscopy. The thermal dye stability was not considered. In a recent study rhodamine 19 dyestuff was evaporated and analysed by absorption spectroscopy [3]. Below $320^{\circ} \mathrm{C}$ the neutral zwitterionic form of rhodamine 19 was found to be stable in the vapor phase. Above $320^{\circ} \mathrm{C}$ rhodamine 19 was observed to disintegrate into the neutral zwitterionic form of 2,7-dimethyl-rhodamine 110 (2[6-amino-3-imino-2,7-dimethyl-3H-xanthen-9-yl] benzoic acid) [4].

In this paper the fluorescence behaviour of rhodamine 19 vapor and of 2,7-dimethyl-rhodamine 110 vapor is investigated. The structural formulae of the neutral zwitterionic forms of these two molecules [5] are displayed in fig. 1. The fluorescence quantum efficiency of rhodamine 19 vapor is found to be very small $\left(q_{\mathrm{v}} \lesssim 0.0025\right)$. The rotational motions of the ethyl-amino groups of rhodamine 19 in the vapor phase at elevated temperatures are probably responsible for the fast radiationless deactivation [5-7]. The fluorescence quantum efficiency of 2,7-dimethylrhodamine 110 vapor is high $\left(q_{\mathrm{v}} \approx 0.63\right)$ and the fluorescence quantum distribution, the stimulated
(I)

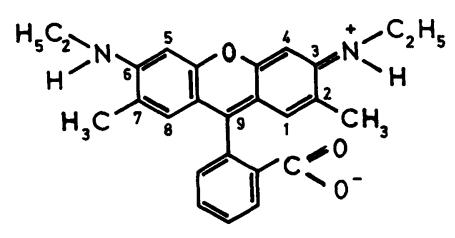

(II)<smiles>[2H]Nc1cc2oc3cc(=[NH+][CH])c(C)cc-3c(-c3ccccc3C(=O)[O-])c2cc1C</smiles>

Fig. 1. Structural formulae of the neutral zwitterionic forms of rhodamine 19 (I), and 2,7-dimethyl-rhodamine 110 (II).

emission cross-section spectrum, and the fluorescence depolarization could be determined.

\section{Experimental}

The vapor cell for the heating of the rhodamine 19 dyestuff is described in ref. [8] (reservoir and cell are made of stainless steel, cell windows consist of sapphire; cell length $l=5 \mathrm{~cm}$ ). The experimental arrangement for the fluorescence studies is depicted in fig. 2. The $457.9 \mathrm{~nm}$ line of an argon ion laser is used 


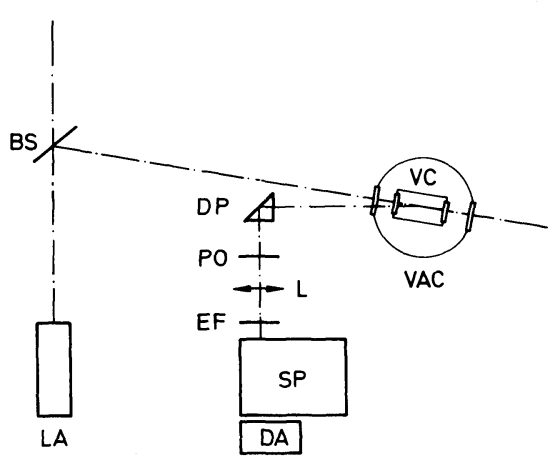

Fig. 2. Experimental arrangement for fluorescence measurement. LA, argon ion laser (Spectra Physics), wavelength $\lambda_{\mathrm{L}}=457.9 \mathrm{~nm}$, average excitation power at vapor cell is approximately $10 \mathrm{~mW}$. BS, beam splitter. VC, vapor cell. VAC, vacuum chamber (for details, see ref. [8]). DP, $90^{\circ}$ deflection prism. PO, dichroitic polarizer. L, lens. EF, dielectric edge filter. SP, grating spectrometer. DA, diode-array system (Tracor DARRS).

for dye vapor excitation (pump power at vapor cell was approximately $10 \mathrm{~mW}$ ). The fluorescence in backward direction is directed to a spectrometer with a $90^{\circ}$ deflection prism and a lens [9]. The scattered pump laser light is discriminated by dielectric edge filters. For fluorescence depolarization studies a dichroitic polarization filter is entered in the fluorescence path and the fluorescence components polarized parallel and perpendicular to the pump pulse polarization are determined. The fluorescence spectra $S_{\mathrm{V}}(\lambda)$ are registered with a diode-array system (Tracor type DARRS system).

\section{Spectroscopic fundamentals}

The absolute fluorescence quantum distribution $E_{\mathrm{V}}(\lambda)$ of the dye vapor is determined by calibrating the fluorescence spectra to the total fluorescence signal, $\int_{e m} S_{R}(\lambda) d \lambda$, of a reference dye solution in the same dye vapor cell. In our experiments a $10^{-7}$ molar solution of rhodamine 19 in methanol was applied (fluorescence quantum efficiency $q_{\mathrm{R}} \approx 0.97[10]$ ).

$E_{\mathrm{V}}(\lambda)$ is given approximately by [9]

$$
\begin{aligned}
& E_{\mathrm{V}}(\lambda)=\frac{S_{\mathrm{V}}(\lambda) / W_{\mathrm{abs}, \mathrm{V}} \Delta \Omega_{\mathrm{V}}}{\int_{\mathrm{em}} S_{\mathrm{R}}(\lambda) \mathrm{d} \lambda / W_{\mathrm{abs}, \mathrm{R}} \Delta \Omega_{\mathrm{R}}} q_{\mathrm{R}} \\
& \quad \approx \frac{S_{\mathrm{V}}(\lambda)}{\sigma_{\mathrm{L}, \mathrm{V}} N_{\mathrm{V}}\left(1-R_{\mathrm{V}}\right)} \frac{\sigma_{\mathrm{L}, \mathrm{R}} N_{\mathrm{R}}\left(1-R_{\mathrm{R}}\right)}{\int_{\mathrm{em}} S_{\mathrm{R}}(\lambda) \mathrm{d} \lambda} \frac{q_{\mathrm{R}}}{n_{\mathrm{F}, \mathrm{R}}^{2}} .
\end{aligned}
$$

The absorbed pump energies in the dye vapor and in the reference dye solution are

$$
\begin{aligned}
& W_{\mathrm{abs}, \mathrm{V}}=W_{\mathrm{L}}\left[1-\exp \left(-\sigma_{\mathrm{L}, \mathrm{V}} N_{\mathrm{V}} l\right)\right]\left(1-R_{\mathrm{V}}\right) \\
& \quad \approx W_{\mathrm{L}} \sigma_{\mathrm{L}, \mathrm{V}} N_{\mathrm{V}} l\left(1-R_{\mathrm{V}}\right) \\
& \text { and } \\
& W_{\mathrm{abs}, \mathrm{R}}=W_{\mathrm{L}}\left[1-\exp \left(-\sigma_{\mathrm{L}, \mathrm{R}} N_{\mathrm{R}} l\right)\right]\left(1-R_{\mathrm{R}}\right) \\
& \quad \approx W_{\mathrm{L}} \sigma_{\mathrm{L}, \mathrm{R}} N_{\mathrm{R}} l\left(1-R_{\mathrm{R}}\right),
\end{aligned}
$$

respectively. $\sigma_{\mathrm{L}, \mathrm{V}}$ and $\sigma_{\mathrm{L}, \mathrm{R}}$ are the absorption cross sections at the pump laser frequency. $N_{\mathrm{V}}$ and $N_{\mathrm{R}}$ are the dye vapor and dye solution number densities.

$R_{\mathrm{V}}=\left(n_{\mathrm{L}, \mathrm{W}}-1\right)^{2} /\left(n_{\mathrm{L}, \mathrm{W}}+1\right)^{2}$

and

$R_{\mathrm{R}}=\left(n_{\mathrm{L}, \mathrm{W}}-n_{\mathrm{L}, \mathrm{R}}\right)^{2} /\left(n_{\mathrm{L}, \mathrm{W}}+n_{\mathrm{L}, \mathrm{R}}\right)^{2}$

are the pump light reflectivities at the sapphire window-dye interfaces $\left(n_{\mathrm{L}, \mathrm{W}}\right.$ is refractive index of sapphire at $\lambda_{\mathrm{L}}, n_{\mathrm{L}, \mathrm{R}}$ is refractive index of reference dye solution at $\lambda_{L}$ ). The ratio of the solid angles of fluorescence acceptance by lens $L$ is

$\Delta \Omega_{\mathrm{V}} / \Delta \Omega_{\mathrm{R}}=n_{\mathrm{F}, \mathrm{R}}^{2} / n_{\mathrm{F}, \mathrm{V}}^{2} \approx n_{\mathrm{F}, \mathrm{R}}^{2}$

$\left(n_{\mathrm{F}, \mathrm{R}}\right.$ is the average refractive index of the reference solution at the wavelength of the fluorescence band; $n_{\mathrm{F}, \mathrm{v}} \approx 1$ ). The spectral characteristics of the elements in the fluorescence path are taken into consideration in the determination of absolute fluorescence quantum distribution $E_{\mathrm{V}}(\lambda)$ (see ref. [9]).

The fluorescence quantum efficiency of the dye vapors is obtained by integration over the fluorescence quantum distribution:

$q_{\mathrm{v}}=\int_{\mathrm{em}} E_{\mathrm{v}}(\lambda) \mathrm{d} \lambda$.

The radiative lifetime, $\tau_{\text {rad, } \mathrm{v}}$, of the dye vapors is determined by application of the Strickler-Berg formula $[11,12]$

$$
\begin{aligned}
& \frac{1}{\tau_{\text {rad, }, \mathrm{V}}}=\frac{8 \pi n_{\mathrm{V}, \mathrm{F}}^{3} c_{0}}{n_{V, A}} \frac{\int_{\mathrm{er}} \lambda E_{\mathrm{V}}(\lambda) \mathrm{d} \lambda}{\int_{\mathrm{em}} E_{\mathrm{V}}(\lambda) \lambda^{4} \mathrm{~d} \lambda} \\
& \times \int_{\text {abs }} \frac{\sigma_{\mathrm{abs}, \mathrm{V}}(\lambda)}{\lambda} \mathrm{d} \lambda .
\end{aligned}
$$

$c_{0}$ is the vacuum light velocity. The refractive indices in the $\mathrm{S}_{1}-\mathrm{S}_{0}$ fluorescence region, $n_{\mathrm{V}, \mathrm{F}}$, and in the $\mathrm{S}_{0^{-}}$ 
$\mathrm{S}_{1}$ absorption region, $n_{\mathrm{V}, \mathrm{A}}$, are practically equal to 1 . $\sigma_{\mathrm{abs}, \mathrm{V}}(\lambda)$ is the absorption cross section of the dye vapor at wavelength $\lambda$. The integrations extend over the $\mathrm{S}_{1}-\mathrm{S}_{0}$ fluorescence band (em) and over the $\mathrm{S}_{0}-\mathrm{S}_{1}$ absorption band (abs).

The stimulated emission spectrum is calculated from the fluorescence quantum distribution $E_{\mathrm{V}}(\lambda)$, the radiative lifetime $\tau_{\mathrm{rad}, \mathrm{v}}$ and the fluorescence quantum efficiency $q_{\mathrm{v}}$ by [13]

$\sigma_{\mathrm{em}, \mathrm{V}}(\lambda)=\frac{\lambda^{4} E_{\mathrm{V}}(\lambda)}{8 \pi \eta_{\mathrm{v}, \mathrm{F}}^{2} c_{0} \tau_{\mathrm{rad}, \mathrm{v}} q_{\mathrm{v}}}$.

The fluorescence depolarization is determined by measuring the components $S_{\mathrm{V}, \|}(\lambda)$ and $S_{\mathrm{V}, \perp}(\lambda)$ of fluorescence light polarized parallel and perpendicular to the pump laser light. The degree of fluorescence polarization, $P$, is given by [14-17]

$$
\begin{aligned}
P & =\frac{\int_{\mathrm{em}} S_{V, \|}(\lambda) \mathrm{d} \lambda-\int_{\mathrm{em}} S_{\mathrm{V}, \perp}(\lambda) \mathrm{d} \lambda}{\int_{\mathrm{em}} S_{\mathrm{V}, \|}(\lambda) \mathrm{d} \lambda+\int_{\mathrm{em}} S_{V, \perp}(\lambda) \mathrm{d} \lambda} \\
& \approx \frac{S_{\mathrm{V}, \|}\left(\lambda_{\max }\right)-S_{\mathrm{V}, \perp}\left(\lambda_{\max }\right)}{S_{\mathrm{V}, \|}\left(\lambda_{\max }\right)+S_{\mathrm{V}, \perp}\left(\lambda_{\max }\right)} .
\end{aligned}
$$

The degree of polarization depends on the reorientation time, $\tau_{\mathrm{or}}$, and on the fluorescence lifetime, $\tau_{\mathrm{F}}$, by $[17-20]$

$\frac{1}{P}-\frac{1}{3}=\left(\frac{1}{P_{0}}-\frac{1}{3}\right)\left(1+\frac{\tau_{\mathrm{F}}}{\tau_{\mathrm{or}}}\right)$.

The reorientation time of a dye molecule is proportional to the viscosity, $\eta$, of the surrounding medium and to the free rotation time, $\tau_{0}$, of the molecule $[21,22]$, i.e.

$\tau_{\text {or }}=\kappa \eta+\tau_{0}$.

For spherical molecules $\kappa$ is given by $\kappa=V / k \vartheta$ $[20,23]$, where $V$ is the hydrodynamic volume of the molecule, $k$ is the Boltzmann constant, and $\vartheta$ is the temperature. The free-rotator time $\tau_{0}$ is given by [21] $\tau_{0}=\frac{2}{9} \pi(I / k \vartheta)^{1 / 2}$ where $I$ is the moment of inertia. $P_{0}$ is the degree of polarization in the absence of reorientation $\left(\tau_{\mathrm{or}} \rightarrow \infty\right)$. For an electric dipole transition with absorption and emission oscillator parallel to one another it is $P_{0}=0.5$ [14-17].

In our experiments the degree of polarization of the reference solution (rhodamine 19 in methanol) is calculated by use of eq. (6) to be $P_{\mathrm{R}}=0.0135$ $\left(\tau_{\mathrm{or}} \approx 100 \mathrm{ps}[24], \tau_{\mathrm{F}}=\tau_{\mathrm{rad}, \mathrm{R}} q_{\mathrm{R}} \approx 4.3 \mathrm{~ns}, P_{0}=0.5\right.$ ).
The degree of polarization $P_{\mathrm{V}}$ of the dye vapor is measured (relative to $P_{\mathrm{R}}$ ) by application of eq. (5). For the vapor the molecular reorientation time (eq. (7)) is equal to the free-rotator time $\tau_{0}(\eta \rightarrow 0)$.

\section{Results}

The measured fluorescence signals are presented in fig. 3. Rhodamine 19 dyestuff was inserted in the reservoir of the vapor cell. The vapor cell temperature, $\vartheta_{\mathrm{C}}$, was kept approximately $15^{\circ} \mathrm{C}$ higher than the reservoir temperature $\vartheta_{\mathrm{R}}$ in order to avoid dye deposition at the sapphire windows of the vapor cell.

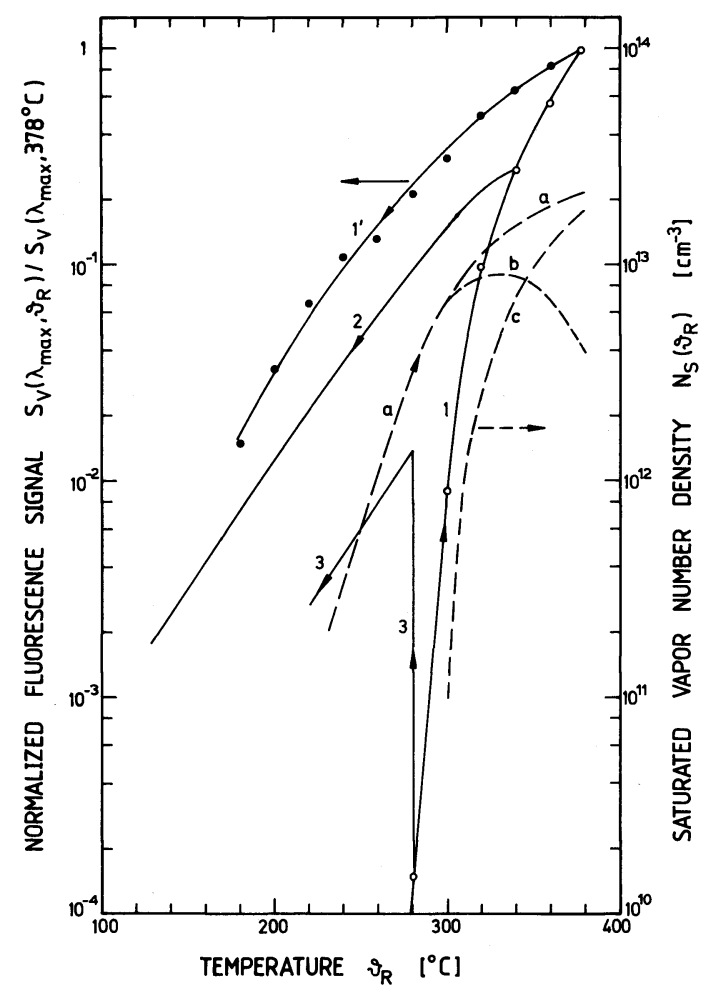

Fig. 3. Temperature dependence of vapor fluorescence signal (solid curves) and of saturated vapor number density (dashed curves, from ref. [3]). (a) Total vapor density. (b) Neutral zwitterionic rhodamine 19 vapor density. (c) Neutral zwitterionic 2,7-dimethyl-rhodamine 110 vapor density. $(1, O)$ Heating of rhodamine 19 dyestuff to $\vartheta_{\mathrm{R}}=378^{\circ} \mathrm{C}$. $\left(1^{\prime}, 0\right)$ Cooling the vapor starting at $\vartheta_{\mathrm{R}}=378^{\circ} \mathrm{C}$. (2) Cooling the vapor after heating to $\vartheta_{\mathrm{R}}=340^{\circ} \mathrm{C}$. (3) Observation of temporal effects by heating to $\vartheta_{R}=280^{\circ} \mathrm{C}$, then remaining at this temperature of a period of 4.5 $\mathrm{h}$ before cooling. 
The dashed curves (a)-(c) of fig. 3 represent vapor densities which were determined in ref. [3]. The curve (a) gives the total vapor density of the neutral zwitterionic rhodamine 19 vapor (curve (b) ) and of the neutral zwitterionic 2,7-dimethyl-rhodamine 110 vapor (curve (c)). In ref. [3] arguments are given that the cationic (protonic) forms of rhodamine 19 and 2,7-dimethyl-rhodamine 110 do not exist in the vapor phase. The lactonic forms of the dyes $[25,26]$ may be present in the vapor phase, but their vapor density contribution is not included in the curves (a) -(c) of fig. 3 [3]. The lactonic forms absorb in the UV spectral region [25,3] and are not excited in our measurements. The disintegration of rhodamine 19 into 2,7-dimethyl-rhodamine 110 is temperature and time dependent. The stainless steel cell walls seem to act catalytic for this decomposition. The curves (a) -(c) were obtained for a heating rate of approximately $2^{\circ} \mathrm{C} / \mathrm{min}$. This heating rate applies also roughly to the fluorescence curve (1).

A comparison of the fluorescence curve (1) (open circles) with the vapor density curve (a) indicates a later onset and a steeper rise of the fluorescence emission. At temperatures $\vartheta_{\mathrm{R}}<300^{\circ} \mathrm{C}$ where practically only rhodamine 19 is in the vapor phase the fluorescence quantum efficiency is very small. At $\vartheta_{R}=300^{\circ} \mathrm{C}$ a total fluorescence quantum efficiency of $q_{\mathrm{v}} \approx 0.0025$ is determined. Since fluorescence contributions of 2,7-dimethyl-rhodamine 110 cannot be excluded at $300^{\circ} \mathrm{C}$, the fluorescence quantum efficiency of rhodamine 19 vapor is thought to be $q_{\mathrm{v}} \lesssim 0.0025$. A fast rotation of the ethylamino groups (see formula (I) of fig. 1) seems to be responsible for the small quantum efficiency [5-7]. For rhodamine 19 in ethanol at room temperature a fluorescence quantum efficiency of $q_{\mathrm{F}}=0.97$ was measured [10]. In liquid solution the solvation seems to hinder the rotation of the ethylamino groups.

At $\vartheta_{\mathrm{R}}=378^{\circ} \mathrm{C}$ (accuracy of temperature measurement is $\pm 5^{\circ} \mathrm{C}$ ) approximately $80 \%$ of the vapor molecules converted to 2,7-dimethyl-rhodamine 110 . Using eqs. (1) and (2) and the fluorescence spectrum at $378^{\circ} \mathrm{C}$ (see fig. 4 below) a fluorescence quantum efficiency of $q_{\mathrm{v}} \approx 0.63$ is determined for 2,7dimethyl-rhodamine 110 vapor. For the commercial dye rhodamine 110 in ethanolic solution at room temperature a fluorescence quantum efficiency of $q_{\mathrm{F}}=0.94$ was measured [10].
The fluorescence curve $\left(1^{\prime}\right)$ (closed circles) is obtained by heating to $378^{\circ} \mathrm{C}$ (curve (1)) and then cooling (rate $\approx-1^{\circ} \mathrm{C} / \mathrm{min}$ ). The vapor density is now given by the thermal equilibrium between 2,7 dimethyl-rhodamine 110 molecules in the vapor phase and at the stainless steel cell walls. The curve $\left(1^{\prime}\right)$ represents the temperature dependence of the 2,7-dimethyl-rhodamine 110 vapor density since the fluorescence quantum efficiency of this dye is thought to be nearly independent of temperature.

The fluorescence curve (2) (no experimental points shown) is measured by heating to $\vartheta_{\mathrm{R}}=340^{\circ} \mathrm{C}$ and then cooling. In the case of curve (3) the cell is heated to $\vartheta_{\mathrm{R}}=280^{\circ} \mathrm{C}$ and then kept at this temperature for $4.5 \mathrm{~h}$. The vertical part of the curve is an indication of the disintegration of rhodamine 19 into 2,7-dimethyl-rhodamine 110 . In cooling, the decay characteristics of the fluorescence curves $\left(1^{\prime}\right),(2)$, and ( 3 ) are the same.

The fluorescence quantum distribution $E_{\mathrm{V}}(\lambda)$ of 2,7-dimethyl-rhodamine 110 vapor (determined from fluorescence spectrum at $378^{\circ} \mathrm{C}$ ) is depicted in fig. 4. The fluorescence maximum is at approximately $515 \mathrm{~nm}$. For rhodamine 19 vapor the fluorescence quantum efficiency is too weak to determine a

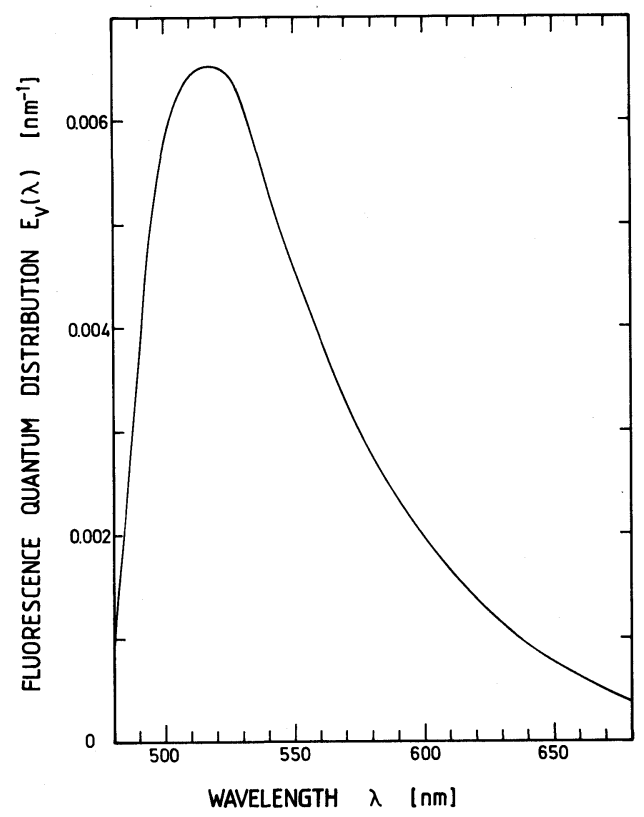

Fig. 4. Fluorescence quantum distribution of 2,7-dimethyl-rhodamine 110 vapor at $\vartheta_{\mathrm{R}}=378^{\circ} \mathrm{C}$. 


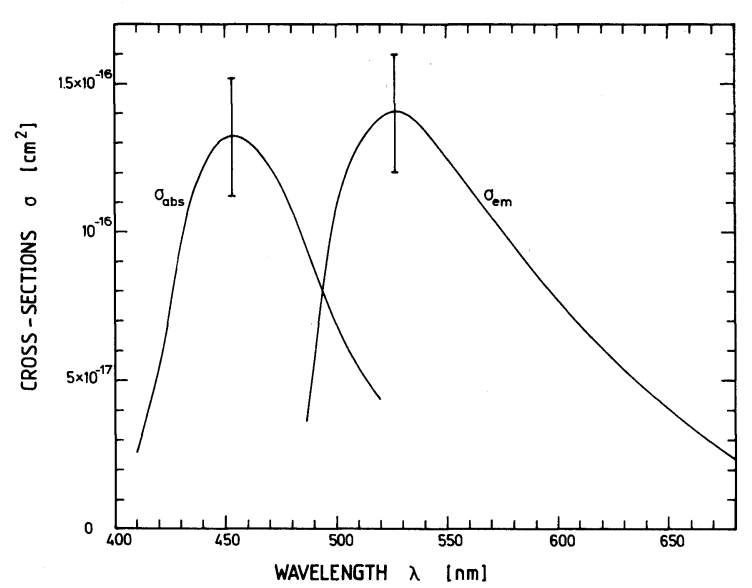

Fig. 5. Absorption cross section spectrum and stimulated emission cross section spectrum of 2,7-dimethyl-rhodamine 110 vapor.

reliable fluorescence quantum distribution.

The absorption cross section spectrum [3] and the stimulated emission cross section spectrum (eq. (4)) of 2,7-dimethyl-rhodamine 110 vapor are shown in fig. 5. A radiative lifetime of $\tau_{\mathrm{rad}, \mathrm{V}} \approx 7 \mathrm{~ns}$ is calculated (eq. (3)). The product of radiative lifetime and fluorescence quantum efficiency gives a fluorescence lifetime of $\tau_{\mathrm{F}, \mathrm{V}}=\tau_{\mathrm{rad}, \mathrm{V}} q_{\mathrm{V}} \approx 4.4 \mathrm{~ns}$.

The degree of fluorescence polarization of the 2,7dimethyl-rhodamine 110 vapor is found to be $P_{\mathrm{V}} \leqslant 0.0045$ (eq. (5)). This polarization value gives a reorientation time of $\tau_{\mathrm{or}} \leqslant 33 \mathrm{ps}$. The result is compatible with an estimate of the free-rotator time of $\tau_{0} \approx 2 \mathrm{ps}\left(\tau_{0}=\frac{2}{9} \pi(I / k \vartheta)^{1 / 2}, I \approx 0.5 m r^{2}, m \approx 6.56 \times\right.$ $\left.10^{-25} \mathrm{~kg}, r \approx 0.4 \mathrm{~nm}, \vartheta \approx 650 \mathrm{~K}\right)$.

\section{Conclusions}

The dye rhodamine 19 was evaporated and the fluorescence behaviour was analysed. While rhodamine 19 vapor has a very small fluorescence quantum efficiency of $q_{\mathrm{v}} \lesssim 0.0025$, the fluorescence quantum efficiency of the disintegration product, 2,7-dimethylrhodamine 110 , is rather high $\left(q_{\mathrm{v}} \approx 0.63\right)$. For this dye vapor the fluorescence quantum distribution, the stimulated emission cross section spectrum and the degree of fluorescence polarization could be determined.

The low vapor density of 2,7-dimethyl-rhodamine
110 hinders the application of this dye as a laser active medium despite the high fluorescence quantum efficiency since the single pass amplification in a cell of $l=5 \mathrm{~cm}$ length at $\vartheta_{\mathrm{R}}=378^{\circ} \mathrm{C}$ is only $G=$ $\exp \left[\sigma_{\mathrm{em}}\left(\lambda_{\max }\right) N_{\mathrm{S}} l\right] \approx 1.013$.

\section{References}

[1] T. Sakurai and H.G. de Winter, J. Appl. Phys. 46 (1975) 875.

[2] R. Pappalardo and S. Ahmed, J. Chem. Phys. 56 (1972) 5135.

[3] H. Weininger, J. Schmidt and A. Penzkofer, Chem. Phys. 130 (1989) 379.

[4] R. Steppel, in: Handbook of Laser Science and Technology, Vol. 1, Lasers and Masers, ed. M.J. Weber (CRC Press, Boca Raton, 1982) pp. 299 ff.

[5] K.H. Drexhage, in Dye Lasers, Topics in Applied Physics, Vol. 1, 2nd Ed., ed. F.P. Schäfer (Springer, Berlin, 1977) p. 144.

[6] W. Rettig, Angew. Chem. 98 (1986) 969.

[7] M. Vogel, Dissertation, Technische Universität Berlin, Berlin, Germany (1987).

[8] J. Schmidt and A. Penzkofer, Chem. Phys. 117 (1987) 265.

[9] A. Penzkofer and W. Leupacher, J. Luminescence 37 (1987) 61.

[10] R. Sens, Dissertation, Universität-Gesamthochschule Siegen, Siegen, FRG (1984).

[11] S.J. Strickler and R.A. Berg, J. Chem. Phys. 37 (1962) 814.

[12] J.B. Birks and D.J. Dyson, Proc. Roy. Soc. A 275 (1963) 135.

[13] O.G. Peterson, J.P. Webb, W.C. McColgin and J.H. Eberly, J. Appl. Phys. 42 (1971) 1917.

[14] Th. Förster, Fluoreszenz Organischer Verbindungen (Vandenhoeck und Ruprecht, Göttingen, 1951).

[15] C.A. Parker, Photoluminescence of Solutions (Elsevier, Amsterdam, 1968).

[16] F. Dörr, Angew. Chemie 78 (1966) 457.

[17] G. Weber, in: Fluorescence and Phosphorescence Analysis, Principles and Applications, ed. D.M. Hercules (Interscience, New York, 1966) p. 217.

[18] R. Memming, Z. Physik. Chem. NF 28 (1961) 168.

[19] A.C. Albrecht, J. Mol. Spectry. 6 (1961) 84.

[20] F. Perrin, Ann. Physique 12 (1929) 238.

[21] D.R. Bauer, J.I. Brauman and R. Pecora, J. Am. Chem. Soc. 98 (1974) 6840.

[22] G.R. Fleming, Chemical Applications of Ultrafast Spectroscopy (Oxford Univ. Press, Oxford, 1986).

[23] A. Einstein, Ann. Physik 19 (1906) 371.

[24] H.E. Lessing and A. von Jena, in: Laser Handbook, Vol. 3, ed. M.L. Stitch (North-Holland, Amsterdam, 1979) pp. 753 ff.

[25] U.K.A. Klein and F.W. Hafner, Chem. Phys. Letters 43 (1976) 141.

[26] F. Barigellitti, Chem. Phys. Letters 140 (1987) 603. 